\title{
Las nuevas clases gramaticales de palabras según la propuesta de la Nueva gramática de la lengua española
}

\author{
Marcela Oyanedel Fernández* \\ José Luis Samaniego Aldazábal ${ }^{* *}$ \\ Academia Chilena de la Lengua y Pontificia Universidad Católica de Chile
}

Una de las labores de la gramática y de los gramáticos es proponer un inventario de las clases gramaticales de palabras de las lenguas históricas y esto porque el número de palabras, en un momento dado, es incontable y, por lo mismo, inmanejable. Asimismo, todas las palabras creadas y por crear en una lengua histórica presentan entre sí determinadas afinidades de comportamiento, lo cual es el fundamento del posible inventario de clases que se puede establecer y, a la vez, una garantía de la estabilidad de la lengua. De modo que se han de entender las clases de palabras como el conjunto de comportamientos regulares que afectan a un grupo de palabras y que por ello permiten agruparlas. Así ha sido como se han establecido distintos inventarios de las clases gramaticales de palabras desde Platón y Aristóteles, pasando por los gramáticos latinos y de la Edad Media y luego, en lo que respeta a nuestra lengua, por Nebrija, Sánchez de las Brozas y

* Para correspondencia, dirigirse a: Marcela Oyanedel Fernández (moyanedf@ uc.cl),Pontificia Universidad Católica de Chile, Facultad de Letras, Departamento de Ciencias del Lenguaje, Campus San Joaquín, Av. Vicuña Mackenna 4860, Santiago, Chile.

** Para correspondencia, dirigirse a: José Luis Samaniego Aldazábal (jsamanie@, uc.cl),Pontificia Universidad Católica de Chile, Facultad de Letras, Departamento de Ciencias del Lenguaje, Campus San Joaquín, Av. Vicuña Mackenna 4860, Santiago, Chile. 
demás gramáticos hasta la doctrina tradicional de la Real Academia Española, que durante muchos años sostuvo las conocidas nueve partes de la oración o nueve clases gramaticales de palabras.

Hoy, la nueva doctrina gramatical de la lengua española de la RAE y de la Asociación de Academias de la Lengua Española (véase RAE y Asale 2009, 2010 y 2011) ofrece una nueva propuesta que nos permitiremos presentar en esta nota. En primer lugar, daremos a conocer que, frente a las nueve clases tradicionales que reconocía la gramática española, distingue ahora nueve clases sintácticas de carácter funcional y siete clases transversales de carácter fundamentalmente semántico. En segundo lugar, delimita con mayor precisión aquellas clases sintácticas que incrementan sus unidades con transferencias procedentes de las clases transversales. De modo que en esta nota nos referiremos a la nueva clasificación que afecta a cinco de las nueve clases sintácticas, de las que daremos a conocer, en primer lugar, su núcleo estable con las innovaciones que se proponen, a lo que agregaremos los flujos procedentes desde las siete clases transversales.

Según esto, las nueve clases gramaticales de carácter tradicional se ordenan en esta nueva propuesta de acuerdo con una mirada que establece distinción entre clases sintácticas, de carácter funcional, y clases transversales, de carácter fundamentalmente semántico. Por ejemplo, los posesivos constituyen una clase semántica por su significado. Ahora bien, los posesivos pueden desempeñar distintas funciones, lo que hace que -de manera transversal- puedan formar parte de más de una clase sintáctica. Así, el posesivo de primera persona singular en mi libro desempeña una función distinta de la que desempeña en el libro mío y en el libro es mío, como también en el mío es nuevo, dependiendo la función del posesivo mío, en este último caso, de si se ha nombrado el sustantivo elidido o no. Distinta también es su función en caso de figurar combinado con el artículo neutro lo, en un ejemplo como lo mío nos pertenece a ambos.

A partir de esta distinción, se reconocen -como decíamos-nueve clases sintácticas y siete clases transversales, con una reordenación diferente de las clases tradicionales. 


\section{LA ACTUAL GRAMÁTICA PROPONE UNA NUEVA CLASIFICACIÓN DE LAS PALABRAS}

\begin{tabular}{|l|l|}
\hline \multicolumn{1}{|c|}{ Clases sintácticas (funcionales) } & Clases transversales (semánticas) \\
\hline Determinantes o determinativos & Demostrativos \\
Sustantivos & Posesivos \\
Adjetivos & Cuantificadores indefinidos \\
Pronombres & Cuantificadores numerales \\
Verbos & Relativos \\
Adverbios & Interrogativos \\
Preposiciones & Exclamativos \\
Conjunciones & \\
Interjecciones & \\
\hline
\end{tabular}

El movimiento entre estos dos tipos de clases de palabras será enfocado en su doble direccionalidad, de lo sintáctico a lo transversal y de lo transversal a lo sintáctico. De modo que las clases transversales se actualizan como clases sintácticas, incrementando estas últimas su caudal al recibir unidades procedentes de las clases transversales. A su vez, las clases sintácticas, al corresponder a determinados usos de las clases transversales, se encontrarán en más de una clase transversal.

\section{CLASES SINTÁCTICAS O FUNCIONALES DE PALABRAS}

De estas solo se considerarán, en esta nota, las cinco siguientes: determinantes, sustantivos, adjetivos, pronombres y adverbios, por ser las que ofrecen a las unidades de las clases transversales la posibilidad de una mayor variedad en su realización funcionaly porque su tratamiento actual presenta mayor innovación. 


\subsection{DeterminANTES O DETERMINATIVOS}

La clase sintáctica de los determinantes, que incluye por derecho propio la subclase de los artículos definidos e indefinidos, se incrementa con los adjetivos determinativos tradicionales.

Por el hecho de anteponerse al sustantivo al cual determinan y actualizan, concretándolo y permitiendo así que este funcione como grupo nominal sujeto de la oración, constituyen los determinantes un mismo paradigma funcional, cuyas unidades pueden sustituirse unas por otras, aun cuando semánticamente pertenezcan a clases o subclases semánticas transversales diferentes. Por ejemplo: la clase transversal de los demostrativos, como este en este libro; de los posesivos, como mi en mi libro; de los cuantificadores indefinidos, como algún en algún libro; de los cuantificadores numerales, como dos en dos libros; de los relativos, como cuyo en el libro cuyas tapas...; de los interrogativos, como cuál en ¿cuál libro?; y de los exclamativos, como qué en iqué libro!

De manera que la nueva clase de los determinantes, además de incluir el artículo por ser esta su función propia, incorpora los tradicionales adjetivos determinativos.

\section{LA PROPIEDAD ESENCIAL DE LOS DETERMINANTES ES ACTUALIZAR AL SUSTANTIVO}

\begin{tabular}{|c|c|c|}
\hline & Demostrativos: & este libro \\
\hline & Posesivos: & mi libro \\
\hline DETERMINANTES & Cuantificadores indefinidos: & algún libro \\
\hline O DETERMINATIVOS & Cuantificadores numerales: & dos libros \\
\hline (el artículo como & Relativos: & ...cuyo libro \\
\hline una subclase: & Interrogativos: & ¿cuál libro? \\
\hline el libro, un libro) & Exclamativos: & ¡qué libro! \\
\hline
\end{tabular}

\subsection{Sustantivos}

A la tradicional clasificación de los sustantivos en comunes y propios, y los comunes en individuales y colectivos, contables y no contables, y concretos y abstractos, se reconocen los de complemento argumental, exigido por su significado, como padre en el padre de Juan e inauguración en la inauguración del puente; los eventivos, que implican una duración y 
un lugar determinados, como reunión en la reunión será breve o concierto en el concierto no durará más de una hora y será en el Teatro Municipal; los cuantificativos, que permiten cuantificar a los sustantivos no contables como litro en un litro de leche o copa en solo una copa de vino; y los clasificativos como especie, clase o tipo en la quinoa es una especie, clase o tipo de legumbre.

A lo anterior se agregan las unidades de ciertas clases transversales que también pueden desempeñarse como sustantivos en determinados usos y las que, incluso, solo se desempeñan como tales. Entre las primeros están los posesivos combinados con el artículo definido neutro lo como mío en lo mío también te pertenece o con otro artículo definido con la condición de que no haya sustantivo elidido como en el mío es más rápido. También, unidades que corresponden unas a las que pueden desempeñarse como sustantivos en determinados usos y otras que solo se desempeñan como tales. Entre estas se incluyen los cuantificadores indefinidos, como es el caso de el todo y la nada o ciertos cuantificadores indefinidos si se combinan con el artículo definido neutro lo, como mucho en lo mucho que me cuesta; y asimismo los cuantificadores numerales, entre los que se distinguen los siguientes: cardinales como seis, par o docena en ella obtuvo un seis o en ella compró un par de zapatos o una docena de huevos; ordinales como segundo o primero en el es alumno de segundo o en lo primero será leer el texto; partitivos como octavo o cuarto en un octavo de aceite o en un cuarto de queso; y múltiplos o multiplicativos como doble o triple en ponle el doble de azúcar o en hubo una triple dificultad.

De manera que en la nueva propuesta, la clase de los sustantivos incluye los tradicionales con una subclasificación de los sustantivos comunes más fina, a la vez que incorpora los posesivos y los cuantificadores indefinidos y numerales cuando desempeñan la función propia del sustantivo. 


\section{LAS NUEVAS CONSIDERACIONES SEMÁNTICAS DEL SUSTANTIVO INCIDEN EN SU COMBINATORIA SINTÁCTICA}

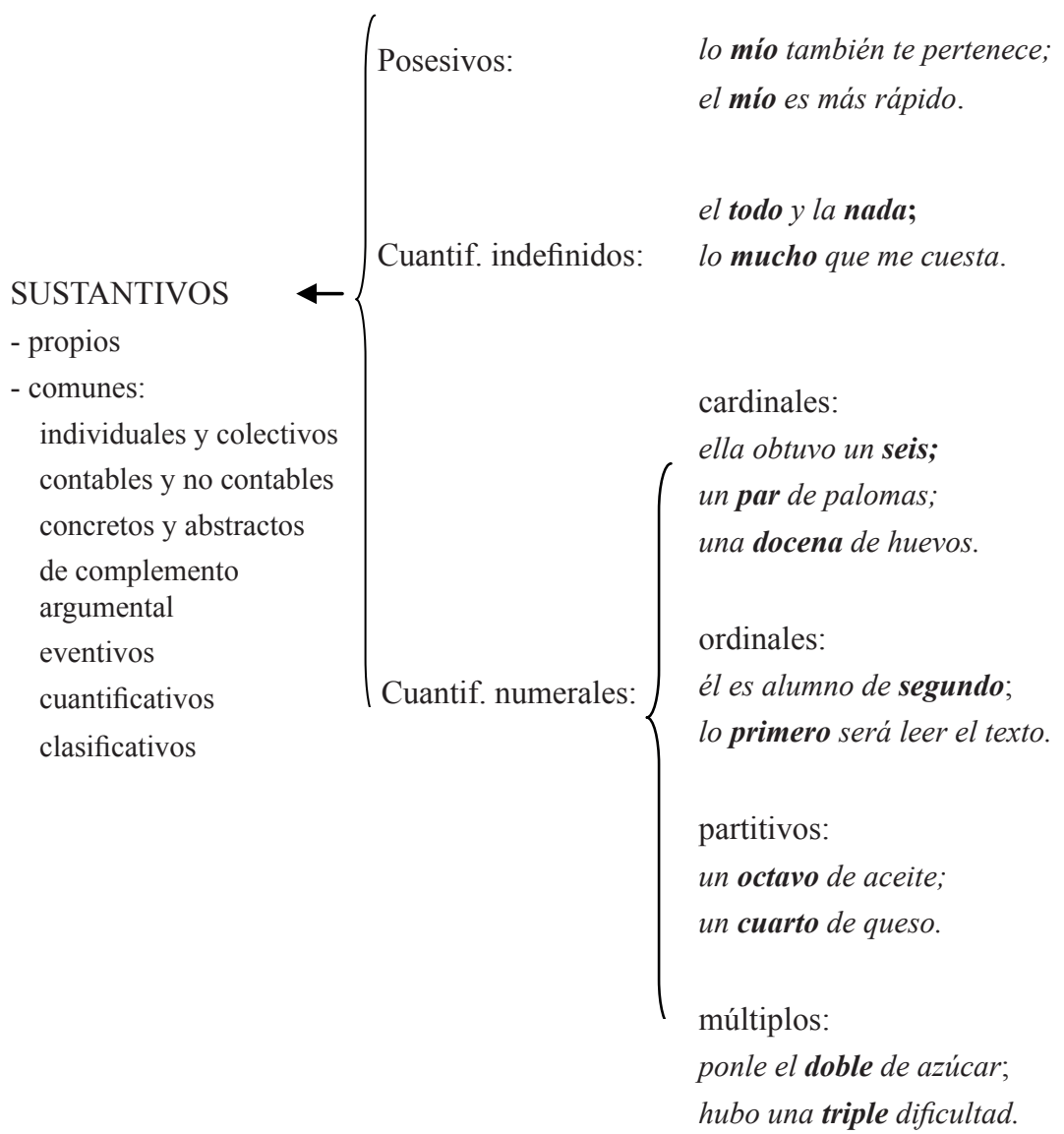

\subsection{Adjetivos}

Esta tercera clase sintáctica presenta importantes diferencias con la tradición, puesto que, si bien incluye los calificativos tradicionales que aportan el significado de cualidad como antigua en puerta antigua, la que es intensificable, como en puerta muy antigua, deja fuera, sin embargo, los adjetivos determinativos, que pasan a la clase de los determinantes ya presentada, esto es, a la primera clase sintáctica. Distingue, en cambio, entre 
los adjetivos calificativos propiamente tales, los adjetivos de relación y los adjetivos adverbiales. Los de relación aportan el significado de propiedad en el sentido del modo de ser, la que no es intensificable, como metálica en puerta metálica, que no puede ser más o menos metálica, ya que es o no es metálica; o como chileno en vino chileno, que no puede ser más o menos chileno, ya que es o no es chileno. Los adjetivos adverbiales, que son adjetivos y, en cuanto tales, concuerdan con el sustantivo, aportan significado de temporalidad, como actual o futuro en la actual o futura directora, o de modalidad, como probable o eventual en el probable o eventual resultado.

A lo anterior se agregan las unidades de ciertas clases transversales que también pueden desempeñarse como adjetivos en determinados usos: los demostrativos clásicos como aquel en el libro aquel, o este en el libro es este y los demostrativos adverbiales de tiempo, modo y lugar, que son adverbios y por lo tanto invariables, como ahí en el libro ahí y acá en los vecinos acá; entonces en el entonces director; así en una respuesta así y en la respuesta es así; los posesivos como el libro mío, el libro es tuyo; los cuantificadores indefinidos como un libro cualquiera, los libros son muchos; los cuantificadores numerales cardinales como los dos libros, los invitados eran tres y los cuantificadores numerales ordinales como el segundo libro, el capitulo cuarto.

De manera que, en la nueva propuesta, los adjetivos, excluidos los determinativos, incluyen los tradicionales calificativos, pero distinguiendo entre los calificativos propiamente tales, los de relación y los adverbiales. A esto se agregan los demostrativos clásicos y los demostrativos adverbiales, los posesivos y los cuantificadores indefinidos y numerales cuando desempeñan la función propia del adjetivo. 


\section{LA CLASE DE LOS ADJETIVOS SE REORDENA EN FORMA NOVEDOSA} Y RECIBE IMPORTANTES APORTES DE LAS CLASES TRANSVERSALES

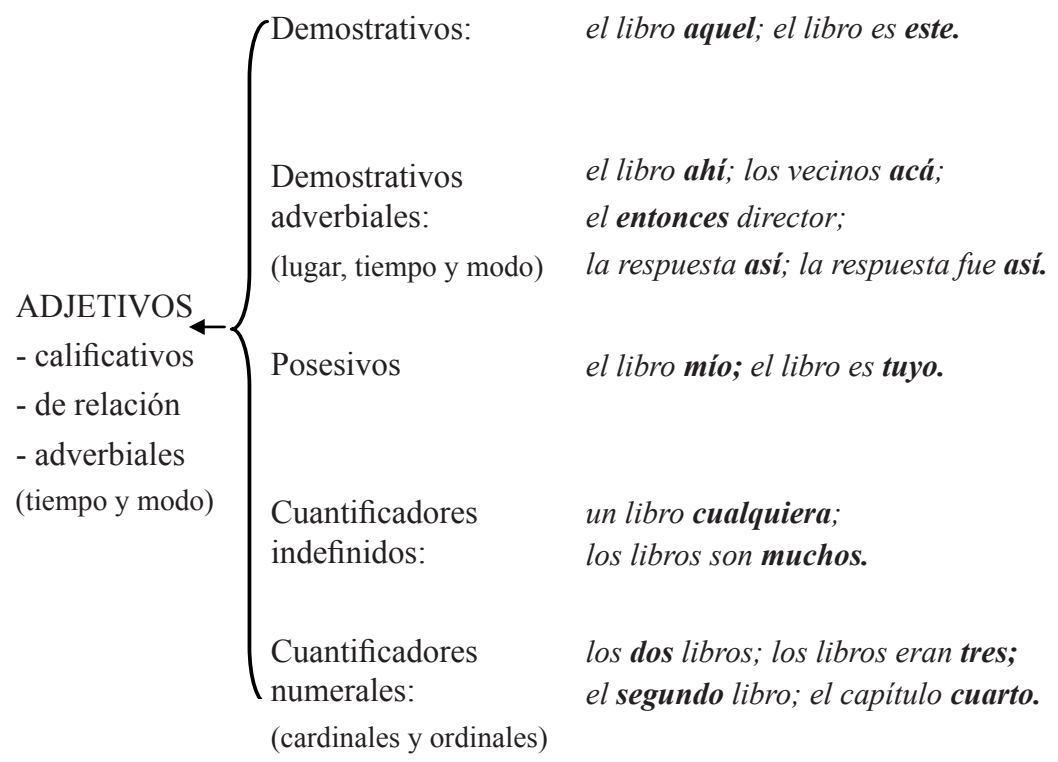

\subsection{PronOmbres}

Constituyen una clase compleja, conformada por la subclase de los pronombres personales, que es clase sintáctica pronominal por excelencia, así ella y lo en ella ya lo estudió, y te y las en te las traeré más tarde. A los personales se agregan ciertas clases transversales, algunas de cuyas unidades solo se desempeñan como pronombres o pueden desempeñarse como tales en determinados usos. Así, las unidades de los demostrativos como en este es más veloz, elijo esa, eso me gusta; las de los cuantificadores indefinidos como en alguien llegó, no tengo nada; las de los cuantificadores numerales cardinales como en tres alcanzaron a llegar; las de los relativos que, el que, quien, el cual y en determinados usos cuanto, como en el libro que leí ..., la persona con quien salí...; la de los interrogativos qué, quién, cuál, cuánto, como en ¿quién asistirá al concierto?, ¿cuántos han visto la película? y las de los exclamativos qué, quién, cuál y cuánto, como en iquién lo diría!, ¡cuántas te comiste! 
También en esta clase sintáctica hay concordancias y diferencias con la tradición. En el primer caso, los demostrativos neutros se consideran siempre pronombres: esto, eso y aquello, como en esto es más interesante que aquello. Actualmente, los demás demostrativos funcionan como pronombres cuando no es posible recuperar por el contexto ni anterior ni posterior, esto es, ni anafórica ni catafóricamente, un sustantivo elidido, como en este es más interesante que ese, sin haber nombrado aquello de lo que se está hablando. Distinto es el caso de este libro es más entretenido que ese, en que ese funciona anafóricamente como determinante del sustantivo libro, que se encuentra elidido, y por lo mismo, el demostrativo no desempeña aquí la función de pronombre.

Con respecto a los cuantificadores indefinidos, su comportamiento sintáctico es muy variado. Por ejemplo, hay algunos que solo son pronombres, así alguien y nadie como en alguien llegó, no vino nadie; otros pueden comportarse como determinantes o como pronombres, así alguno y ninguno como en ningún socio, ninguno quiso hablar; algo y nada son pronombres como en ellos sabían algo, no dijeron nada interesante o adverbios como en algo débil, nada hábil o, incluso, sustantivos como el todo y la nada.

Los relativos que, el que, quien, el cual son siempre pronombres dentro de la oración subordinada relativa que introducen, así que en el libro que leí...; el que en el libro de ese autor, el que lei el año pasado...; quien en Pedro, quien acaba de llegar,...; el cual en el libro de ese autor, el cual quedó finalista en el concurso,... El relativo cuanto, en cambio, además de poder desempeñarse como determinante en llévate cuantos libros quepan en tu bolso, puede funcionar como pronombre en llévate cuantos puedas. Pero en de estos libros, llévate cuantos puedas, cuantos funciona como determinante del sustantivo libros que se encuentra elidido.

Los interrogativos qué, quién, cuál y cuánto cuando funcionan como pronombres, como en ¿quién conoce la respuesta?, ¿cuál te gusta?

Los exclamativos qué, quién, cuánto cuando funcionan como pronombres, como en iquién llegó!, ¡icuánto comiste!

De manera que, en la nueva propuesta, los pronombres, además, de incluir los personales por ser esta su función propia, incorporan los demostrativos, cuantificadores indefinidos y numerales, los relativos, interrogativos y exclamativos, cuando desempeñan la función propia del pronombre. 


\section{LOS PERSONALES SON SOLO PRONOMBRES Y LO SON POR EXCELENCIA}

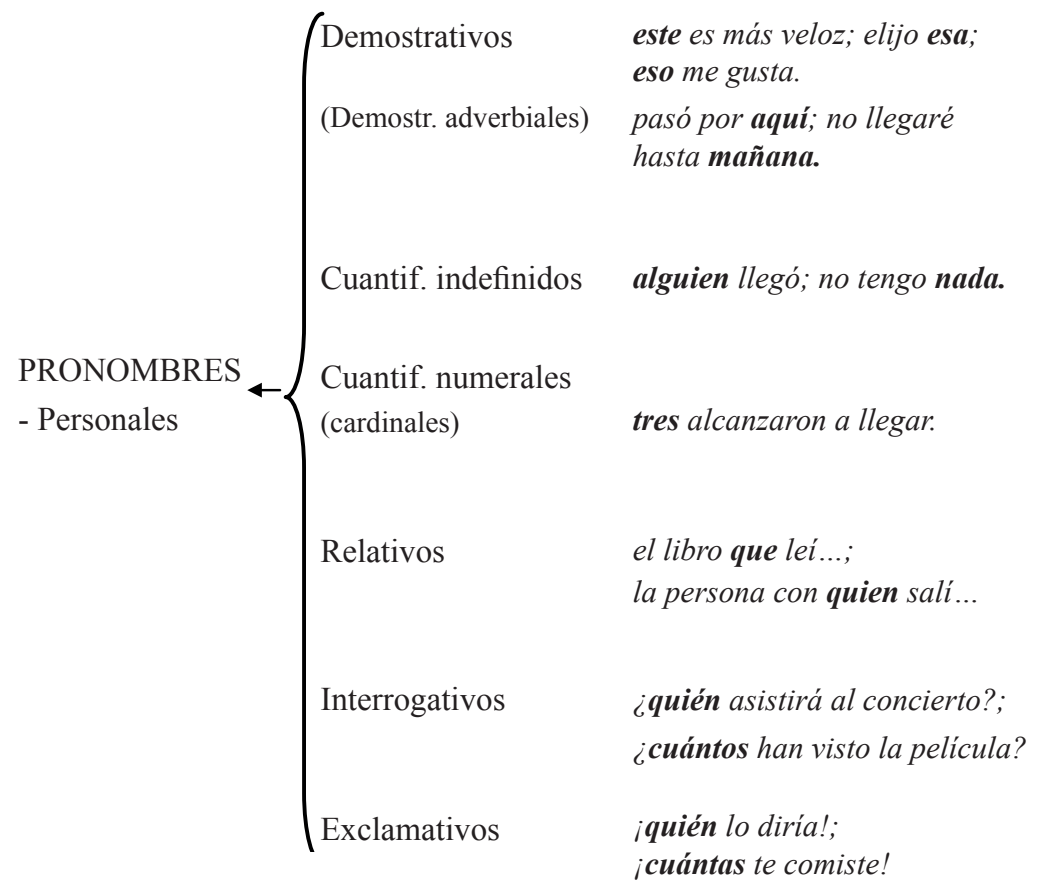

\subsection{Adverbios}

Los adverbios constituyen una clase de palabras tan heterogénea que incluso se ha considerado que, más que una clase, constituyen un conjunto que incluye muchas regularidades individuales, razón que explica el hecho de que surjan tantos criterios distintos para intentar clasificarlos.

Actualmente se clasifican los adverbios según cuatro criterios:

a. según su significado: lugar, tiempo, aspecto, modo o manera, cantidad, afirmación, negación y duda o posibilidad. Se trata de las unidades ya conocidas, salvo las que corresponden a los adverbios de aspecto como son ya, todavía, aún, que más que una noción temporal expresan el aspecto perfectivo o imperfectivo del proceso significado por el verbo. 


\section{b. según su estructura morfológica:}

- simples: aquí, cerca, entonces, siempre, así, bien; o complejos (locuciones adverbiales): de repente, por si acaso, sin duda.

- primitivos: bien, mal, lejos; o derivados: creados sobre la pauta adjetivo terminado en $-a$ si es de dos terminaciones como tranquila, más el sufijo -mente (tranquilamente) o adjetivo pleno si es de una, como leal, más el sufijo -mente (lealmente).

- Por recategorización: los llamados adverbios adjetivales, que vienen de la recategorización de algunos adjetivos calificativos (canta bajo, come sano, juega limpio, se portaron genial).

Solo son adverbios los provenientes de adjetivos que se comportan de manera invariable:

Las golondrinas vuelan bajo (adverbio).

Las golondrinas vuelan bajas (adjetivo que funciona como predicativo).

c. según su naturaleza gramatical, se dividen en:

1. léxicos, que forman clase abierta, como en temprano, altiro, de repente $y$ adverbios en -mente.

2. gramaticales, que forman paradigmas cerrados con siete subclases:

a) demostrativos:

lugar: ahí, aquí, allí, acá, allá.

tiempo: entonces, ahora, hoy, ayer, anteayer, mañana, pasado mañana.

modo: así.

b) identificativos o referenciales:

de ubicación: delante/detrás, encima/debajo, dentro/fuera de dirección: adelante/atrás, arriba/abajo, adentro/afuera

En el español peninsular se construyen con complementos únicamente los adverbios del grupo (a): está delante de la casa, encima de la escalera, dentro de la estación.

En el español americano, los adverbios de dirección también se usan con valor de ubicación y, por lo mismo, admiten construirse con complementos: está arriba de la mesa, está afuera de la casa, está atrás de la cocina. 
c) cuantificadores indefinidos: corresponden a los adverbios de cantidad: algo, mucho, demasiado, suficiente.

d) relativos: cuando, cuanto, como, donde.

e) interrogativos: cuándo, cuánto, cómo, dónde.

f) exclamativos: cuándo, cuánto, cómo, dónde, qué.

g) focales: incluso, aun, hasta, ni siquiera, solo: Solo Juan lo dijo una vez; Juan solo lo dijo una vez, Juan lo dijo solo una vez.

\section{d. según su incidencia sintáctica:}

1. adverbios argumentales versus adjuntos de un verbo (circunstanciales): Los adverbios argumentales son exigidos por un verbo como parte de su significación y solo excepcionalmente pueden suprimirse: pon aquí la chaqueta; se portó estupendamente; mide poco; dura demasiado.

Los adverbios adjuntos o circunstanciales no son exigidos semánticamente por el verbo: habla bien o habla demasiado; viven lejos o viven mal.

2. adverbios atributivos: los adverbios de modo pueden desempeñar la función sintáctica de atributo (modificador de los verbos copulativos ser, estar y parecer, y de verbos semicopulativos como andar, quedar, lucir): está así, quedó así; o de complemento predicativo (modificador directo de un verbo no copulativo, a la vez que modificador indirecto de sujeto o de complemento directo): llegamos bien, la encontré mal.

3. adverbios modificadores de adjetivos y de adverbios:

a) de adjetivos: muy tontos, menos atrevidos, nada fácil;

b) de adverbios: harto lejos, demasiado temprano, bastante bien.

4. adverbios de tópico y de punto de vista: aquí, sí lo saben; mañana, ya veremos si nos juntamos; artísticamente, es digno; lingüísticamente, eso es correcto.

5. adverbios del enunciado: habló sinceramente, escribe bien.

6. adverbios de la enunciación: a) referidos al hablante como en sinceramente, no lo creo; b) dirigidos al oyente como en sinceramente, ¿quién lo rompió? 
A lo anterior se agregan ciertas clases transversales que también pueden desempeñarse como adverbios en determinados usos. Las unidades léxicas (palabras simples y locuciones) correspondientes a estas clases ya fueron mencionadas en los paradigmas correspondientes a los distintos criterios con que se clasifica actualmente el adverbio. No obstante, los indicaremos por el hecho de corresponder a clases transversales. Se trata de los cuantificadores indefinidos que modifican a un verbo: habló mucho, poco, bastante, demasiado, etc.; que modifican a un adjetivo: vive muy, poco, bastante, demasiado tranquila, etc.; o que modifican a un adverbio: llegaste muy, bastante, demasiado, algo lejos, etc..; de los relativos: cuando, cuanto, donde, como (la casa donde vivo...., la forma como te comportas..., el año cuando viajaste a Europa); de los interrogativos cuándo, cuánto, dónde, cómo (¿dónde vives?; ¿cuándo viajaste?; ¿cómo te llamas?; ¿cuánto mide?); de los exclamativos cuánto (cuán), cuándo, dónde, cómo, qué:;dónde te vengo a encontrar, ;cuándo me lo iba a imaginar!, ;cómo no te iba a saludar!, ;cuánto me alegra volver a verte!, iqué lejos vives!

De manera que, en la nueva propuesta, los adverbios, además de incluir las subclases tradicionales con una subclasificación más fina, agregan de las clases transversales los cuantificadores indefinidos y los relativos, interrogativos y exclamativos, cuando desempeñan la función propia del adverbio. 


\section{LA CLASE DE LOS ADVERBIOS INCORPORA UNA AMPLIA VARIEDAD FUNCIONAL A TRAVÉS DE NUEVAS MIRADAS}

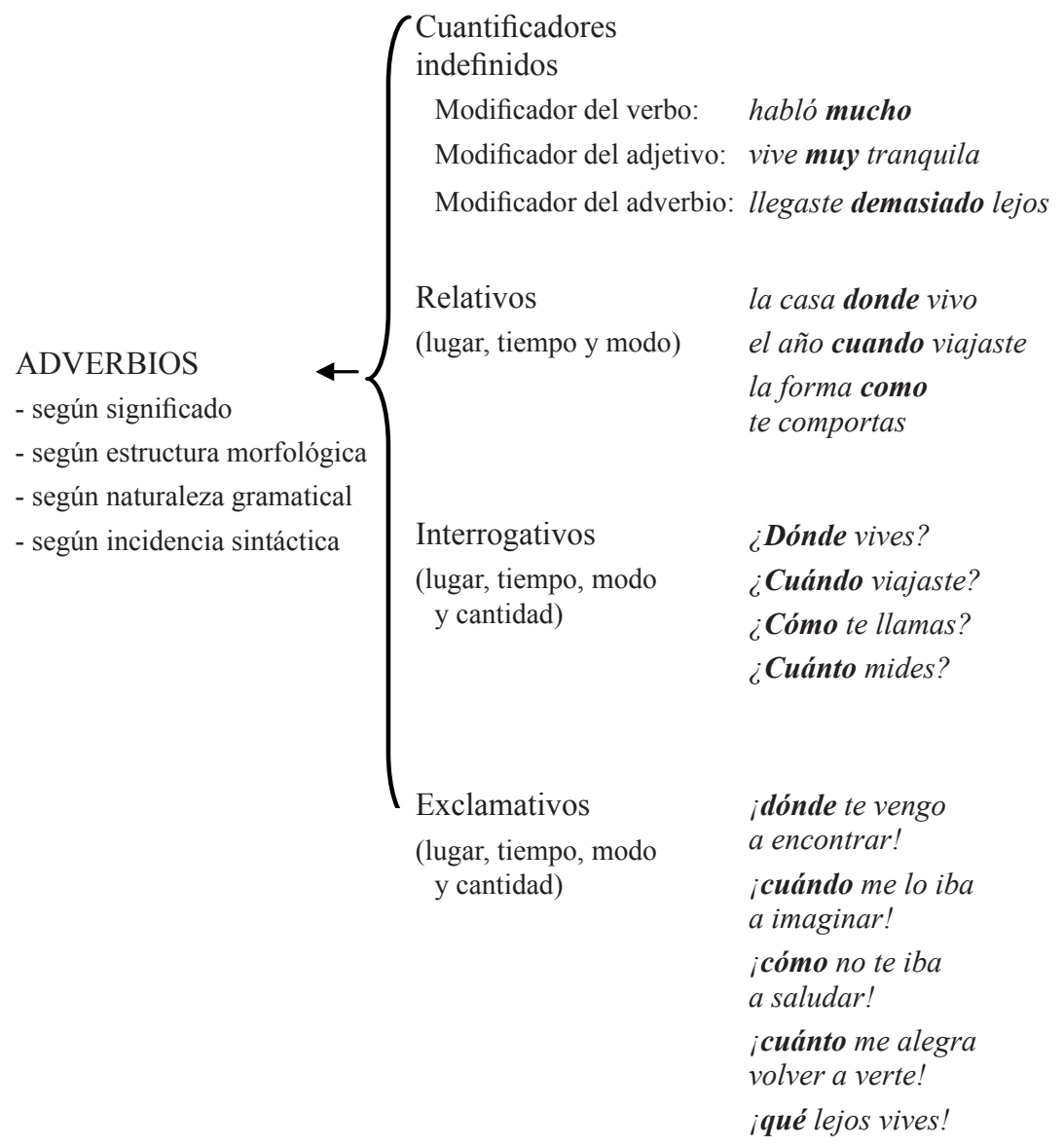

\section{CLASES TRANSVERSALES DE PALABRAS}

Algunas de las propiedades de naturaleza semántica que permiten clasificar las palabras en clases transversales constituyen rasgos cruzados y, por lo tanto, pueden aparecer en distintas clases sintácticas. Estos rasgos 
constituyen las llamadas clases transversales de palabras, cuyas unidades pueden pertenecer a varias categorías o clases sintácticas. Por ejemplo, los posesivos pueden desempeñarse como determinantes, sustantivos y adjetivos; los demostrativos, cuantificadores indefinidos, relativos e interrogativos, como determinantes, adjetivos, pronombres y adverbios; y los cuantificadores numerales, como determinantes, adjetivos y sustantivos.

\subsection{LOS DEMOSTRATIVOS}

Desempeñan función deíctica en la comunicación, por cuanto señalan o sitúan en el espacio, el tiempo, la manera y la cantidad, con interpretación ocasional. Entre estos se encuentran los clásicos demostrativos este, ese, aquel y sus variantes de género y número, incluyendo las formas neutras esto, eso, aquello, a las que se agregan los casos especiales tal y dicho. También se incluyen ciertos adverbios que presentan valor deíctico, tales como así, ahí, entonces y tanto (comió así, está ahí, entonces llegará, leyó tanto), que reciben el nombre de demostrativos adverbiales.

\section{DEMOSTRATIVOS ADVERBIALES}

de lugar: aquí, ahí, allí, acá, allá;

de tiempo: entonces, ahora, hoy, ayer, anteayer, mañana, pasado mañana; de modo: asi;

de cantidad: tanto, así.

El hecho de que tengan interpretación ocasional significa que se llenan de sentido según la situación espacial, temporal, modal o, incluso, cuantitativa. Por ejemplo, si alguien dice estoy aquí, aquí se interpreta como el lugar en que se encuentra esa persona en ese momento, lugar que naturalmente puede variar. En ese sentido es ocasional.

Los demostrativos pueden desempeñarse como miembros de las siguientes clases sintácticas:

determinantes: este libro es más entretenido que ese (libro).

$>$ adjetivos: el libro ese; el libro es ese; el ahora director; el compañero acá; la respuesta fue así; el resultado estuvo mal.

> pronombres: esto es recomendable; este es recomendable; pasó por aquí; no llegará hasta mañana.

adverbios: aquí compré el libro; mañana llegará mi hermano; comí tanto. 
Funcionan como adverbio cuando modifican a verbos no copulativos ni semicopulativos, esto es, que no sean los verbos ser, estar, parecer o verbos del tipo de andar, quedar y lucir; o cuando estos no funcionan como tales.

\subsection{LOS POSESIVOS}

Los posesivos incluyen las siguientes formas: mío, tuyo, suyo, nuestro, vuestro, sus variantes de género y número y las formas apocopadas $m i, t u$, $s u . .$. con sus respectivas variantes de número. A lo anterior hay que agregar el relativo cuyo y sus variantes de género y número. En el caso de lo mío, lo tuyo, etc., se trata de los sustantivos mío, tuyo, suyo, etc., esto es, de los posesivos sustantivados por la presencia del determinante artículo neutro $l o$, o de otro artículo definido en caso de no haber un sustantivo elidido, como en el mío es mejor.

Los posesivos pueden corresponder a las siguientes clases sintácticas:

$>$ determinantes: $\boldsymbol{t} \boldsymbol{u}$ libro es entretenido.

$>$ adjetivos: el libro tuyo; el libro es tuyo; tu libro es más entretenido que el (libro) mío.

$>$ sustantivos: lo tuyo nos pertenece a ambos; la mía puede abrir cualquier puerta.

\subsection{LOS CUANTIFICADORES INDEFINIDOS}

Pueden asumir las siguientes clases sintácticas:

$>$ determinantes: algunos libros; tú tienes muchos libros y yo, algunos (libros).

$>$ adjetivos: los apuntes fueron pocos.

$>$ pronombres: nadie vino; alguien llegó atrasado; asistieron pocos.

> sustantivo: lo mucho que me cuesta este ejercicio.

$>$ adverbio: comió demasiado; muy contento; bastante lejos; bastante poco.

\subsection{LOS CUANTIFICADORES NUMERALES}

\begin{tabular}{|c|c|}
\hline $\begin{array}{l}\text { Cardinales: } \\
\text { Ordinales : } \\
\text { Fraccionarios o partitivos: } \\
\text { Multiplicativos o múltiplos: }\end{array}$ & $\begin{array}{l}\text { dos, tres, cinco, treintaicinco, etc. } \\
\text { segundo, tercero, quinto, trigesimoquinto, etc. } \\
\text { medio, tercio, octavo, onceavo, etc. } \\
\text { doble, triple, cuádruple, etc. }\end{array}$ \\
\hline
\end{tabular}


Los cuantificadores numerales pueden asumir las siguientes clases sintácticas:

$>$ determinantes:

- numerales cardinales: tres libros;

- numerales fraccionarios o partitivos: media naranja es suficiente.

$>$ adjetivos:

- numerales cardinales: los tres libros; las hojas eran tres;

- numerales ordinales: el tercer libro; el libro tercero; el piso catorce (en que el cardinal asume el valor de ordinal);

- numerales fraccionarios o partitivos: esta media naranja; la tercera parte; la doceava parte;

- numerales múltiplos o multiplicativos: la doble vuelta de llave; un triple salto mortal.

sustantivos:

- numerales cardinales: ella obtuvo un seis; pero también los cardinales siguientes: par, docena, decena, centena o centenar, millar, millón, billón, ciento y mil: varios cientos de personas; una docena de huevos duros;

- numerales ordinales: son alumnos de segundo; lo primero será consultar a un experto;

- numerales fraccionarios o partitivos: un cuarto de harina; un doceavo de los habitantes;

- numerales múltiplos o multiplicativos: el doble de comida.

\subsection{LOS RELATIVOS}

Pueden asumir las siguientes clases sintácticas:

$>$ determinantes: Solamente cuyo y cuanto. Así en la casa cuya puerta está siempre abierta; leyó cuantos libros tuvo a su alcance; de esos libros, leyó cuantos (libros) tuvo a su alcance.

$>$ pronombres: las formas son que, el que, quien, el cual, cuanto: el libro que leí; la persona con quien conversé; quien canta su mal espanta; el libro de cuentos que me prestaste, el que leí ayer; el lápiz con el cual dibujé el afiche; trae cuantos puedas comprar; trae todo cuanto puedas. 
$>$ adverbios: donde, cuando, como y cuanto: la casa donde vivió Neruda; el año cuando el hombre llegó a la luna; la manera como contestaste; recoge tanto cuanto puedas; donde vives queda lejos; cuando regreses será un gran día.

\subsection{LOS INTERROGATIVOS}

Pueden asumir las siguientes clases sintácticas:

$>$ determinantes: qué, quién, cuál, cuánto: ¿qué película trajiste; ¿cuántos libros leiste?; ¿cuál lugar prefieres?; ¿cuántas manzanas comiste; de las manzanas que te traje ¿cuántas (manzanas) comiste?

$>$ pronombres: qué, quién, cuál, cuánto: ¿qué trajiste; ¿quién leyó el libro?; ¿cuál prefieres?; ¿cuántas comiste?

$>$ adverbios: dónde, cuándo cómo, cuánto, qué tan: ¿dónde vives?; ¿cuándo regresaste?; ¿cómo te sientes?; ¿cuánto te demoraste?; ¿cuán lejos te propones llegar en la vida?; ¿qué tan lejos queda el cine?

\subsection{LOS EXCLAMATIVOS}

Pueden asumir las siguientes clases sintácticas:

> determinantes: qué, cuánto: ¡qué sorpresa!; ¡cuánta nieve!

$>$ pronombres: qué, quién, cuál, cuánto: ¡en qué estamos metidos!; ¡quién pudiera alcanzarte!; ¡cuál no sería mi sorpresa!; ¡cuántos vinieron a la fiesta!

$>$ adverbios: dónde, cómo, cuánto, cuándo, qué, cuán: ¡dónde vinimos a encontrarnos!; ; cómo me emocionaron tus palabras!; ;cuánto me emocionaron tus palabras!; ; desde cuándo que no te veía!; ;qué contenta se puso al verte!; ; cuán contenta se puso al verte! 


\section{PALABRAS FINALES}

La clasificación de las palabras que propone la nueva gramática académica, más allá de algunas carencias e imprecisiones, implica, a nuestro juicio, un avance significativo. En primer lugar, porque hay un mayor reconocimiento del funcionamiento real y actual de las palabras. Así, por ejemplo, por el hecho de sacar de la clase de los adjetivos los tradicionales adjetivos determinativos y agruparlos con los artículos bajo una misma clase funcional, la de los determinantes o determinativos, nos permite explicar con mayor coherencia la necesidad de actualización que presenta el sustantivo cuando funciona como grupo nominal sujeto de la oración, actualización que se cubre indistintamente, además del artículo, con cualquiera de los nuevos determinantes, más allá del aporte semántico propio de cada clase. En segundo lugar, es un avance porque establece distinciones más finas en algunas clasificaciones, como es el caso, entre otros, de los sustantivos eventivos, clasificativos y cuantificadores; asimismo en el caso de los adverbios aspectuales, focales, de tópico y punto de vista y de la enunciación; esto nos permite a nosotros profesores explicarnos y explicar a otros, especialmente a aprendices de español, ciertos comportamientos combinatorios que son clave de nuestra lengua, como por ejemplo el hecho de que para los sustantivos eventivos usemos el verbo ser: ¿Dónde es el concierto? Es en el teatro Municipal y, en cambio, el verbo estar para la pregunta y respuesta siguientes: ¿Dónde está el teatro Municipal? Está en la calle Mayor. Es también un avance, porque se hace cargo de manera bastante coherente del problema que presentaban ciertas zonas poco precisas y hasta polémicas como es el caso de los posesivos en combinación con artículo. Además por considerar todo el caudal que incrementan las clases transversales a determinadas clases funcionales, según nos hemos referido. Finalmente, cabe destacar que la propuesta académica $-\mathrm{y}$ no solo en este tema del inventario de clases-incorpora, en buena medida, avances logrados por las ciencias del lenguaje en las últimas décadas.

\section{REFERENCIAS BIBLIOGRÁFICAS}

RAE y Asale. 2009. Nueva gramática de la lengua española. Madrid: Espasa.

RAE y Asale. 2010. Nueva gramática de la lengua española. Manual. Madrid: Espasa.

RAE y Asale. 2011. Nueva gramática básica de la lengua española. Madrid: Espasa. 